\title{
Consideraciones para el dimensionamiento de soluciones de sistemas de gestión de edificios
}

Considerations for sizing of the solutions for the building management system

Sergio Andrés Rossells Lovera

sergiorossells@gmail.com

Código ORCID: 0000-0003-1665-4183

\footnotetext{
Articulo aceptado en septiembre 2020

- Arbitrado en octubre 2020

$<$ Publicado en enero 202
}

\section{Universidad Católica Boliviana La Paz - Bolivia}

Resumen

Si bien los Sistemas de Gestión de Edificios (BMS) generan ventajas competitivas, su diseño e implementación son complejos. Esta investigación provee las consideraciones mínimas necesarias para dimensionar una solución BMS. El desarrollo del proceso tiene un alcance descriptivo. La investigación fue validada mediante consulta a expertos e involucra la utilización de herramientas oficiales de Lenovo, Johnson Controls y propias. Como resultado de la investigación se propone un proceso para el dimensionamiento de soluciones que contempla los componentes de hardware, software y servicios mínimos para generar una propuesta de diseño. Se concluye que el proceso propuesto puede asistir y reducir tiempos en las tareas de diseño de soluciones integrales para BMS.

Palabras clave: Edificio inteligente; Sistema de administración de edificios; Solución BMS; soluciones para edificios inteligentes; arquitectura BMS

\begin{abstract}
Although Building Management Systems (BMS) generate competitive advantages, their design and implementation are complex. This research provides the minimum considerations necessary to size a BMS solution. The development of the process has a descriptive scope. The research was validated by consulting experts and involves the use of official tools from Lenovo, Johnson Controls and their own. As a result of the research, a process for the dimensioning of solutions is proposed that considers the hardware components, software and minimum services to generate a design proposal. It is concluded that the proposed process can assist and reduce time in the design tasks of integral solutions for BMS..
\end{abstract}

Palabras clave: Smart building; building management system; BMS solution; Smart building solutions; BMS architecture 


\section{INTRODUCCIÓN}

Tal como expone Gökçe (2012), los Sistemas de Gestión de Edificios (Building Management System, a partir de ahora "BMS" por sus siglas en inglés) son sistemas informáticos que monitorean y controlan los subsistemas técnicos y de servicios de uno o más edificios. Individualmente proveen información en línea, histórica y tendencias sobre los subsistemas. Según Van Hoof (2018), los principales motivos para la inversión en BMS son la reducción de costos en la operación de instalaciones.

El consumo de energía de un edificio puede ser reducido con un BMS como explica Nguyen (2012), la correlación de información de consumo energético en edificios públicos o comerciales puede tener un gran ahorro. Según Gökçe (2012), el 40\% del consumo de energía a nivel mundial corresponde al uso en edificios, tras la investigación de Osama (2018) se reconoce que en los Estados Unidos los edificios consumen $41 \%$ de energía del país. De manera similar en 2014, en la Unión Europea se reconoció que el consumo de edificios fue el $37 \%$ de la energía total, Osama (2018). En la Unión Europea según la Fundación Giner de los Ríos (2016) ya se consideran a los sistemas BMS como una herramienta para optimizar los recursos energéticos de los edificios.

Esta investigación tiene por objetivo proponer un proceso que incluya las consideraciones mínimas necesarias para dimensionar adecuadamente una solución BMS, contempla el dimensionamiento de componentes de hardware, software, servicios e interacción de subsistemas. La implementación de soluciones integrales de BMS requiere un equipo multidisciplinario capacitado en infraestructura de tecnología, manejo de datos, ciberseguridad, BMS y dispositivos de control (Vasques, 2011; Microsoff, 2019).

En la bibliografía revisada se encuentran documentos aislados o de propósito específico. Sin embargo, se ha detectado la necesidad de documentar sobre como dimensionar los componentes que comprenden una solución de BMS y las consideraciones específicas para el dimensionamiento del sistema integral. Este estudio es de gran utilidad para los profesionales que tienen la responsabilidad de dimensionar las soluciones de esta naturaleza. El proceso de dimensionamiento correcto evitará costos adicionales en la implementación, aporta una base de información para la ejecución del proyecto y explica los alcances del sistema.

El documento también se justifica por el aporte práctico y por los beneficios económicos que puede representar para empresas e industrias a nivel internacional, es una oportunidad de ahorro en los costos operativos de un edificio o complejo de edificios. Asimismo, debe considerarse la posibilidad de la extracción de la información del BMS hacia un sistema de inteligencia de negocios. Esto agregará más puntos de vista al sistema de inteligencia incrementando así el 
valor de la información del sistema de apoyo para la toma de decisiones.

Con estos antecedentes, la investigación se constituye en una contribución teórica y práctica para todo profesional que requiera implementar soluciones BMS, el desafío es la recopilación, sistematización y adecuación de la teoría vigente sobre el objeto de estudio. El rigor científico con que se investigó y redactó los hallazgos dan valor teórico a estas páginas. A continuación, se presenta el marco de referencia del estudio. Como explica Astesana y Medina (2016), las soluciones BMS requieren hacer una introducción de los sistemas involucrados, para luego concentrar todos en uno principal que realiza el monitoreo y gestión de todos los sistemas técnicos del edificio. Así, se optimiza la funcionalidad de cada sector del predio o edificio y se logra mayor aprovechamiento de los recursos y servicios. Perry (2017), propone determinar el uso y utilidad que los subsectores empresariales dan y reciben al adoptar tecnologías inteligentes. Asimismo, plantea establecer si esta utilidad es relevante como para influir verdaderamente en el sector de la construcción comercial.

Por su parte, Osama (2018), contribuye definiendo todos los factores que intervienen en el proceso de diseño de un edificio inteligente y modelo conceptual que ayuda a reducir el dióxido de carbono emisiones. Otro aporte importante en este ámbito es el que presenta Van Hoof (2018), cuando examina el estado del contexto de los espacios inteligentes y analizar lo que piensan los tomadores de decisiones al considerar y adoptar la tecnología de espacios inteligentes.

Applied Risk (2019), aporta con un enfoque crítico, cuestiona la utilidad y plantea la necesidad de determinar el nivel de vulnerables de los BMS. Asimismo, establece el papel de los proveedores de BMS en relación con los principios de seguridad por diseño. El estudio de Applied Risk finaliza resaltando la importancia de definir las responsabilidades de los usuarios finales de las soluciones BMS.

\section{METODOLOGÍA}

Se trata de un estudio de alcance descriptivo y corte transversal, que presenta un proceso para el dimensionamiento de soluciones BMS. El proceso se desarrolla en cuatro etapas: 1) consideraciones generales, relevamiento de variables, 3) diseño de arquitectura y 4) consenso.

La etapa 1 demandó tratamiento cualitativo de las variables monitoreo y control, programación de equipos, registro histórico, análisis de datos e infraestructura tecnológica. Por otra parte, se trabajó desde una perspectiva cuantitativa a lo largo del desarrollo de las etapas 2, 3 y 4, las herramientas empleados son provistas por los fabricantes de hardware y software (Lenovo Data Center Solution Configurator, Johnson Controls), herramientas independientes (Iris), tablas 
de cálculo (Excel), herramientas de dibujo asistido (AutoCAD®, Visioß) y herramientas propias para este propósito.

Para comprobar la pertinencia del proceso propuesto se aplicó la técnica de validación por juicio de expertos, el procedimiento consistió en consultar el dictamen de especialistas de marcas de cada componente del sistema y de empresas fabricantes de los componentes. Gracias a la orientación brindada por DATEC, empresa líder en el rubro de soluciones tecnológicas de Bolivia, se seleccionaron cuatro profesionales con especialidades en servidores y almacenamiento, redes de datos, BMS y arquitectura de soluciones de tecnología. El relevamiento de variables y diseño de arquitecturas consideró como referencia las recomendaciones de los fabricantes para el uso adecuado del hardware y software. Los especialistas a su vez acceden a las herramientas de los fabricantes y cuentan con el apoyo directo del fabricante para la elaboración del diseño de cada componente.

\section{RESULTADOS}

En este apartado se presenta el proceso propuesto para el correcto dimensionamiento de soluciones BMS y se ejemplifica la aplicación de estos preceptos en un BMS para un hospital. La Figura 1 muestra en resumen las cuatro etapas y la información relevante que se procura en cada etapa. Este proceso puede iterar para realizar ajustes de presupuesto y cambios en el requerimiento original.

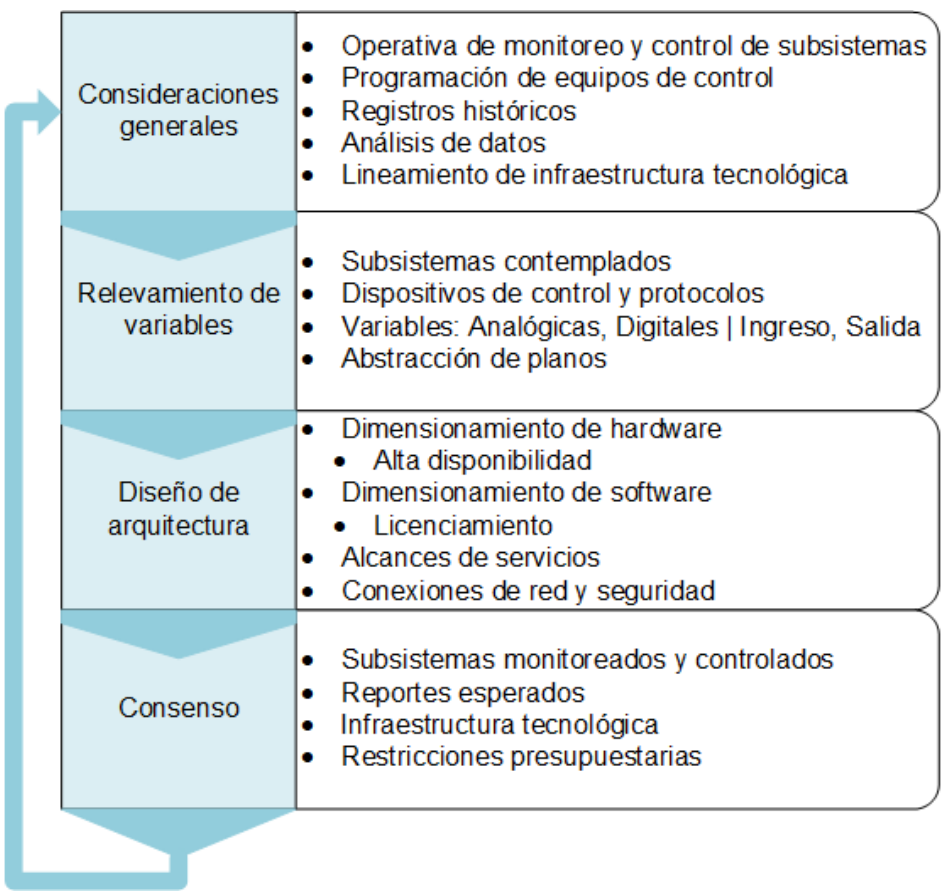

Figura 1. Etapas del proceso de dimensionamiento. 


\section{Ełapa 1. Consideraciones generales}

Dado que la implementación de una solución integral de BMS requiere habilidades multidisciplinarias en infraestructura de servidores, infraestructura de redes, bases de datos, virtualización, ciberseguridad, el propio BMS y los dispositivos de control que este vaya a gestionar. Se debe contar con los recursos capacitados en cada área para asesorar el dimensionamiento de cada componente que interactúe con la solución. Antes de empezar el proceso de dimensionamiento, el equipo a cargo deberá tomar en cuenta consideraciones generales relacionadas con los roles de control y monitoreo, programación de equipos de control, registro histórico, análisis de datos e infraestructura tecnológica. Entender a cabalidad los requerimientos ahorrará tiempo y esfuerzo más adelante, ya que se evitará readecuaciones innecesarias durante la fase de implementación. Se describen a detalle las consideraciones que deberán ser analizadas.

\section{Monitoreo y control}

Se deben considerar los roles una o más estaciones de monitoreo y control que permitan la administración de alarmas y eventos del edificio en tiempo real. El tamaño, complejidad y operativa del edificio, podrán demandar diversos roles de operadores y administradores para la gestión del edificio. También, se debe considerar la posibilidad de que la operativa requiere el enmascaramiento automático o manual de información sensible en el desarrollo de las vistas para mantener la confidencialidad de la información. La segmentación de permisos y vistas de estos roles pueden impactar en la cantidad de estaciones de monitoreo, creación de vistas de los sistemas y el licenciamiento de estos dependiendo del tipo de acceso que se requiera (cliente pesado, cliente web, cliente móvil).

El monitoreo de subsistemas no solamente comprende la lectura y almacén de datos si no también proporciona información para la ejecución y programación de tareas de mantenimiento preventivo a los sistemas. La entrada de datos de subsistemas solamente monitoreados también puede aportar a la programación de comportamientos de sistemas controlados del edificio. Por ejemplo, tomando variables de entrada de CCTV para accionar dispositivos de iluminación.

\section{Programación de equipos.}

La información obtenida de los sistemas del edificio proporcionará la entrada para la configuración del BMS. De acuerdo con el tamaño, tipo y propósito del edificio, se deben definir comportamientos en función a las tareas operativas del edificio, ejecutar acciones sobre equipamiento, automatizar tareas en base a reglas o comportamientos, preparar flujos ante eventos imprevistos 0 periódicos. Asimismo, el diseño de estas tareas demandará horas de capacitación en el sistema para los operadores del 
sistema de acuerdo con los privilegios de cada rol que se defina (ej.: operador, supervisor, administrador).

\section{Registro histórico}

La información histórica del BMS debe ser almacenada y retenida. La información histórica del estado de variables brindará una base para posterior análisis de datos. El almacenamiento de acciones de operadores, alarmas y eventos brindarán evidencias para fines de auditoría y control. El almacenamiento de la información operativa brinda la base para el diagnóstico de fallas, análisis de tendencias, aseguramiento de calidad, cumplimiento regulatorio y contabilidad. La capacidad de almacenamiento se verá afectada directamente por el volumen de información (transacciones) administrada por el BMS y el tiempo de retención (años) requerido de esta información. El tiempo de retención de datos puede estar sujeto a regulaciones del sector.

\section{Análisis de datos}

Las herramientas de análisis de datos son esenciales para la toma de decisiones de los operadores y administradores. Las capacidades más allá de filtros, búsquedas y clasificaciones de alarmas y otros atributos pueden requerir el desarrollo de reportes avanzados. El desarrollo de reportes avanzados puede impactar en más horas de servicio e incluso en licenciamiento adicional. Es recomendable considerar capacidades para la extracción de datos, análisis estadísticos y generación de informes en esta etapa o para futuro. Esto puede comprender la integración a otros sistemas para mejor aprovechamiento de la información. Por ejemplo, integración de datos hacia el sistema contable para la imputación de gastos de energía por área. Parte de estas herramientas de reportería avanzada y análisis pueden ser provistas por el mismo fabricante de BMS.

\section{Infraestructura tecnológica}

El dimensionamiento de los componentes de hardware viene ligado directamente al volumen de información estos van a procesar y al nivel de disponibilidad requerido. El BMS es un sistema crítico del edificio. La falta de disponibilidad puede interrumpir la operación normal de los sistemas controlados. La cantidad de núcleos, memoria, redes y almacenamiento se pueden estimar de acuerdo con métricas del proveedor de software para BMS. La inclusión de una plataforma de virtualización brindará alta disponibilidad al sistema. La capa de virtualización incrementará la cantidad de núcleos, memoria y almacenamiento. La capacidad requerida para virtualización dependerá de las recomendaciones del proveedor del hipervisor. La solución de BMS deberá estar homologada por el fabricante para la virtualización. El almacenamiento también debe contemplar 
componentes redundantes para garantizar la alta disponibilidad a través de arquitecturas tradicionales (DAS, SAN o Convergente) o a través de nodos Hiperconvergentes en el que cada nodo aporta capacidades de procesamiento y almacenamiento. Si se requiere infraestructura nueva (proyecto greenfield) para alojar al BMS, se debe tomar en cuenta la experiencia disponible de los administradores de sistemas para la gestión de la plataforma. En caso de falta de experiencia, las capacitaciones requeridas para aminorar la brecha. Durante el dimensionamiento de la infraestructura tecnológica también deben considerarse las licencias de software necesarias para habilitar la infraestructura. Asimismo, los contratos de soporte/suscripción de los fabricantes de software, hardware, hipervisores, sistemas operativos, bases de datos y conexiones de clientes/dispositivos.

También debe definirse el acuerdo de nivel de servicio que se requiere para la atención de incidentes del sistema, mantenimiento preventivo y horas de soporte.

\section{Etapa 2. Relevamiento de variables}

El BMS puede integrarse con diversos subsistemas del edificio como: almacenamiento de combustible, sistema de agua caliente, CCTV, climatización, control de acceso, sistemas de evacuación, distribución eléctrica, distribución de gases, hidrosanitario, transporte vertical, iluminación, etc. La cantidad y tipo de variables dependerán de los subsistemas que se decidan integrar al BMS. Cada subsistema puede tener variables digitales y analógicas de entrada y de salida.

Es necesario contar con la participación de los instaladores de estos sistemas para la correcta consideración de variables y validación de protocolos de comunicación.

Los subsistemas que tengan variables de entrada permitirán conocer y medir estados (variables de monitoreo), en caso de existir variables de salida será posible definir escalas y estados (variables de control).

El correcto relevamiento de las variables es determinante para lograr un adecuado dimensionamiento de la solución BMS. El procedimiento implica conocer las características de los subsistemas y la configuración de sus variables, para posteriormente realizar la abstracción de sus componentes en el sistema. Es recomendable considerar la participación del ingeniero de cada subsistema para la obtención de los datos consolidados y validados. Se debe obtener la cantidad de variables digitales y analógicas y cuales de ellas son de monitoreo y control. Asimismo, los protocolos utilizados por los dispositivos de control. En esta etapa, también debe participar el equipo de redes de datos para validar la cantidad de puertos libres en switches de acceso para los dispositivos de control. Al mismo tiempo, se debe definir un esquema de ciber seguridad para proteger de la 
mejor manera la transmisión de información entre dispositivos a través de la red de datos como el manejo de contraseñas seguras, mantenimientos programados para actualización de dispositivos, reglas de firewall, segmentación de redes por propósito, etc.

Las horas de servicio requeridas para la configuración de subsistemas en el BMS será impactada por el tipo y cantidad de variables, conexión a dispositivos de control, abstracción de dispositivos de campo, configuración de los sistemas, configuración de reglas y comportamientos.

En esta etapa, también se debe obtener planos para conocer la disposición de los ambientes del edificio. La abstracción física de las áreas de edificio o complejo también impactarán en las horas de servicio.

\section{Etapa 3. Diseño de arquitectura}

Según se presenta en la Figura 2, toda arquitectura del BMS considera tres (3)

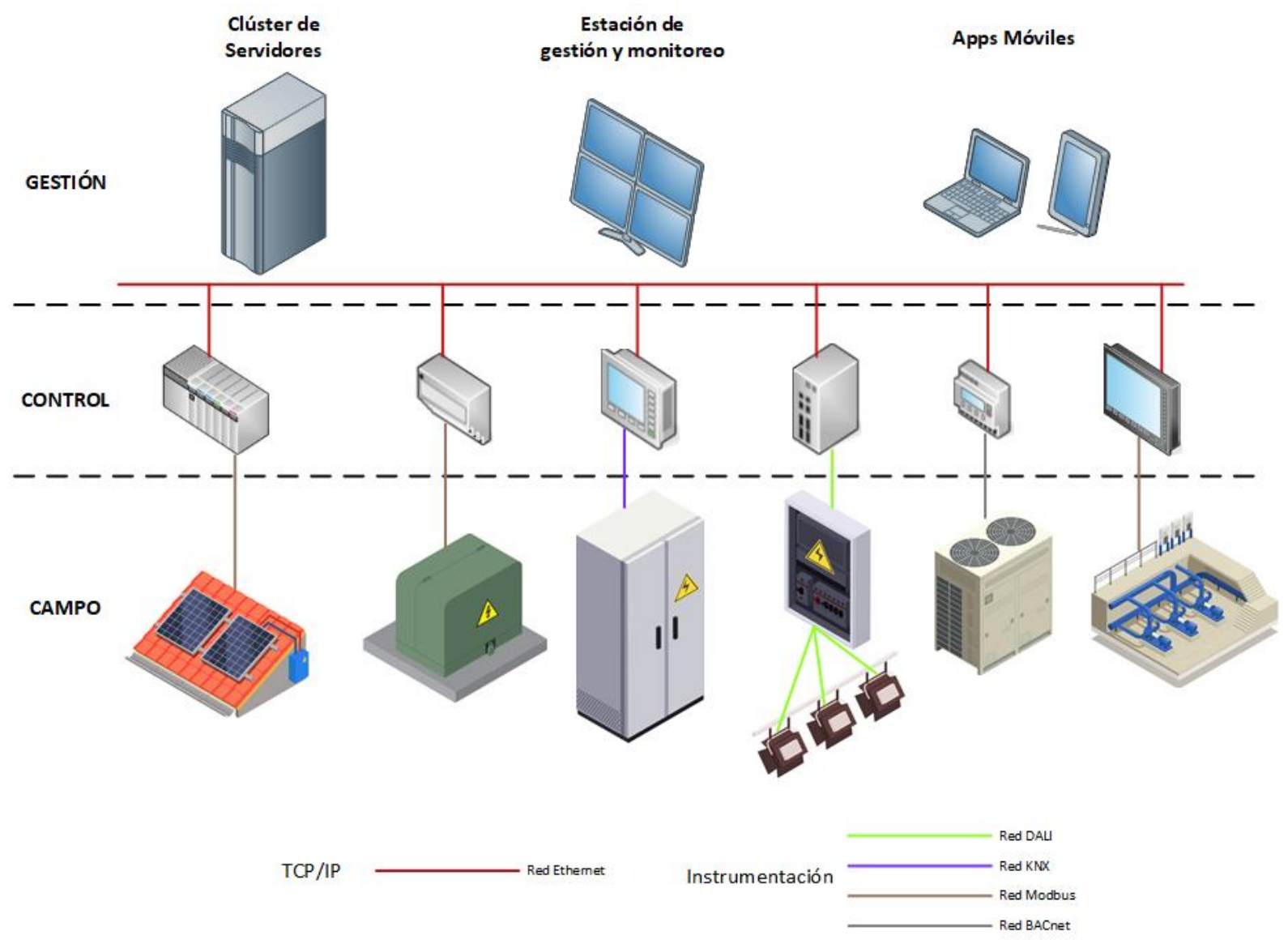

Figura 2. Arquitectura general de un Sistema BMS 
El gráfico 2 muestra la arquitectura general del funcionamiento de un sistema BMS, que consiste en la interrelación entre: capa de gestión, capa de dispositivos de control y capa de dispositivos de campo. A continuación, se explica a detalle el objetivo de cada capa.

Capa 1, plataforma de gestión. Comprende los servidores o clúster que alojan a los servicios del BMS. Se encuentran las estaciones de monitoreo y control para la gestión del edificio. A este nivel, también se realiza la integración a otros sistemas (Video Management System, Control de Acceso, Directorio Activo, Bases de Datos, Herramientas de análisis, etc.). Las comunicaciones en esta capa se realizan a través de TCP/IP. En este nivel se abstraen los subsistemas del edificio para su gestión, monitoreo y control. Se realizan las programaciones de horarios, comportamientos del edificio, reglas y condiciones. Asimismo, la generación de vistas para monitoreo en línea y reportes históricos de tendencias, eventos y alarmas.

Capa 2, control de procesos. Esta capa comprende los dispositivos de control y monitoreo específicos a los subsistemas del edificio. Estos pueden comprender detección y supresión de incendios, transporte vertical, distribución y generación eléctrica, sistemas de confort, iluminación, persianas, calidad del aire, control de acceso, CCTV, intrusión, etc.

Los dispositivos de control son propios de cada subsistema (eléctrico, confort, transporte, etc.). El dimensionamiento, distribución e instalación de ellos está sujeto al diseño cada sistema al que pertenecen. Es decir, son provistos y mantenidos por los instaladores/mantenedores del sistema. Estos dispositivos deben contar con un puerto en la red de datos (TCP/IP) para comunicación con el BMS y con conexiones hacia los dispositivos de campo formando la red de instrumentación. La red de instrumentación puede utilizar protocolos de comunicación industriales con los dispositivos de campo (Ej.: Modbus, BACnet, KNX, DALI, OPC, SNMP, etc.). Los dispositivos de control son la pasarela de interconexión entre la red de datos y la red de instrumentación.

Capa 3, dispositivos de campo. Controlados directamente desde la capa de control, los dispositivos de campo obtienen información (sensores de: estado, temperatura, luz, movimiento, etc.) y controlan mecanismos de los subsistemas (válvulas, interruptores, actuadores, motores, etc.). En esta capa se debe se realiza la conexión con los componentes propios de los subsistemas técnicos del edificio. La provisión, instalación y configuración de ellos están sujetos al diseño cada subsistema. Cada uno de estos de elementos será abstraído en el BMS.

\section{Dimensionamiento de hardware $y$ software}

Para

que

el

correcto dimensionamiento de la solución, se debe considerar los componentes de 
conexión de redes y servidores, según se muestra en la Figura 3 como referencia.

En la Figura 3 se presenta el diagrama de conexión a nivel de red de datos. Es importante considerar redundancia de conexiones de red en los servidores y equipos de red para incrementar la disponibilidad ante fallas o mantenimientos. Las controladoras de campo, una vez conectadas al servidor, envían lecturas (monitoreo) hacia el BMS y reciben instrucciones (control) desde el BMS. Dependiendo el proveedor del sistema, las controladoras también pueden disponer puertos para conexión redundante. También se debe considerar que la alimentación eléctrica de las controladoras pueda ser a través de PoE (Power over Ethernet). La cantidad de dispositivos conectados y alimentados a la red de acceso impactará en el dimensionamiento de switches de acceso. Este proceso no contempla el dimensionamiento de switches de acceso. Sin embargo, esta información relevada en esta etapa puede disparar adecuaciones previas al sistema de red de datos.

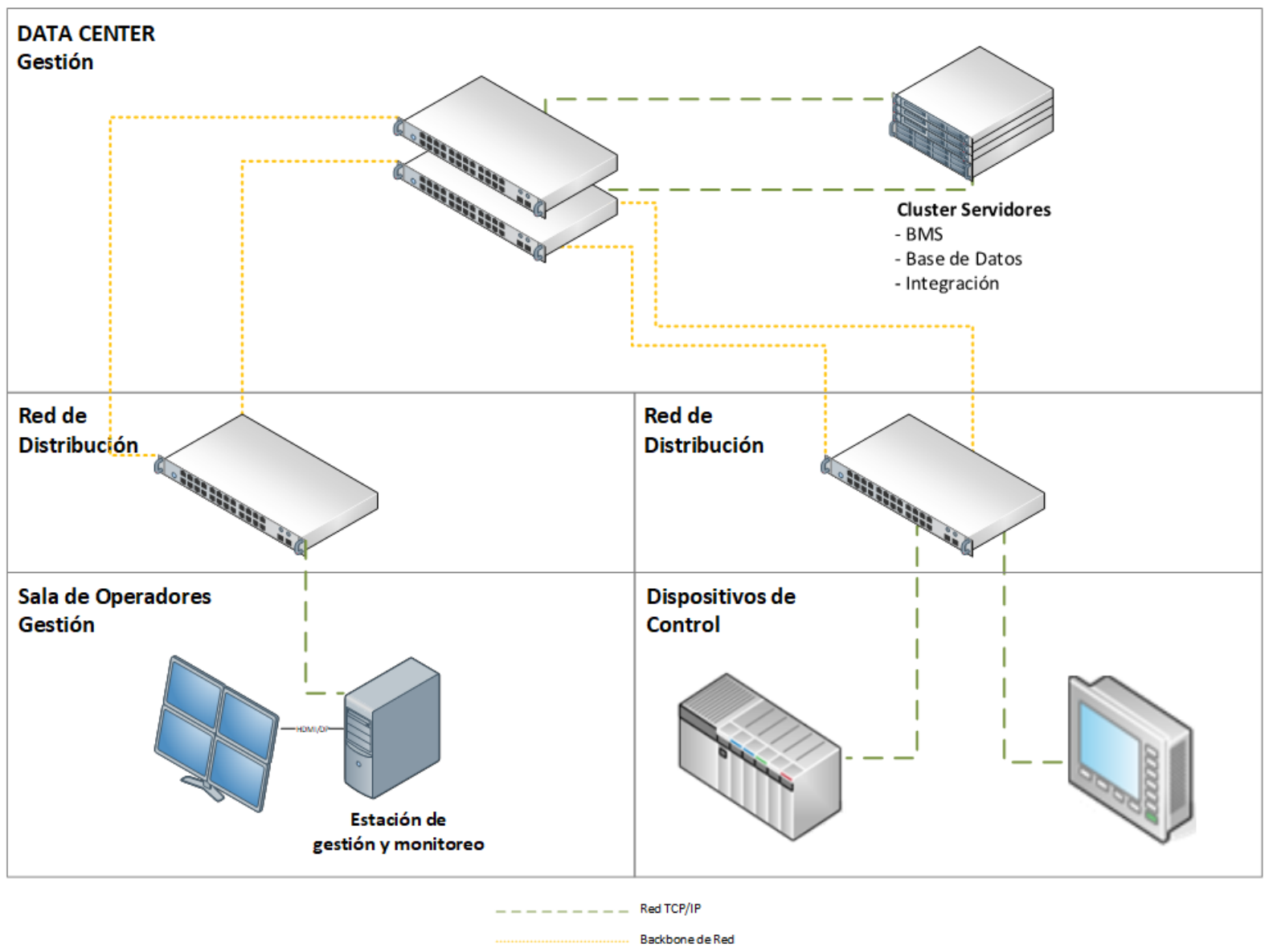

Figura 3. Conexiones de red de datos. 
El dimensionamiento del equipamiento de servidores deberá realizarse de acuerdo con la cantidad de variables consideradas, según las recomendaciones de los fabricantes y las tablas de dimensionamiento de hardware. Con estas métricas es posible dimensionar las especificaciones requeridas para el sistema. La provisión de infraestructura puede requerir un clúster nuevo o habilitación de recursos sobre infraestructura existente.

En el caso de que el proyecto demande infraestructura nueva, se recomienda un clúster de servidores con propósito específico. El clúster habilita capacidades para alta disponibilidad de los servidores del sistema BMS, bases de datos y otros servicios de integración (control de acceso, directorio, etc.). Es necesario dimensionar las especificaciones de los servidores físicos considerando un porcentaje de crecimiento.

Adelante se presenta un diagrama de alto nivel de un clúster de servidores contemplando conexiones redundantes en la red de datos (LAN) y la red de almacenamiento (SAN) (ver Figura 4).

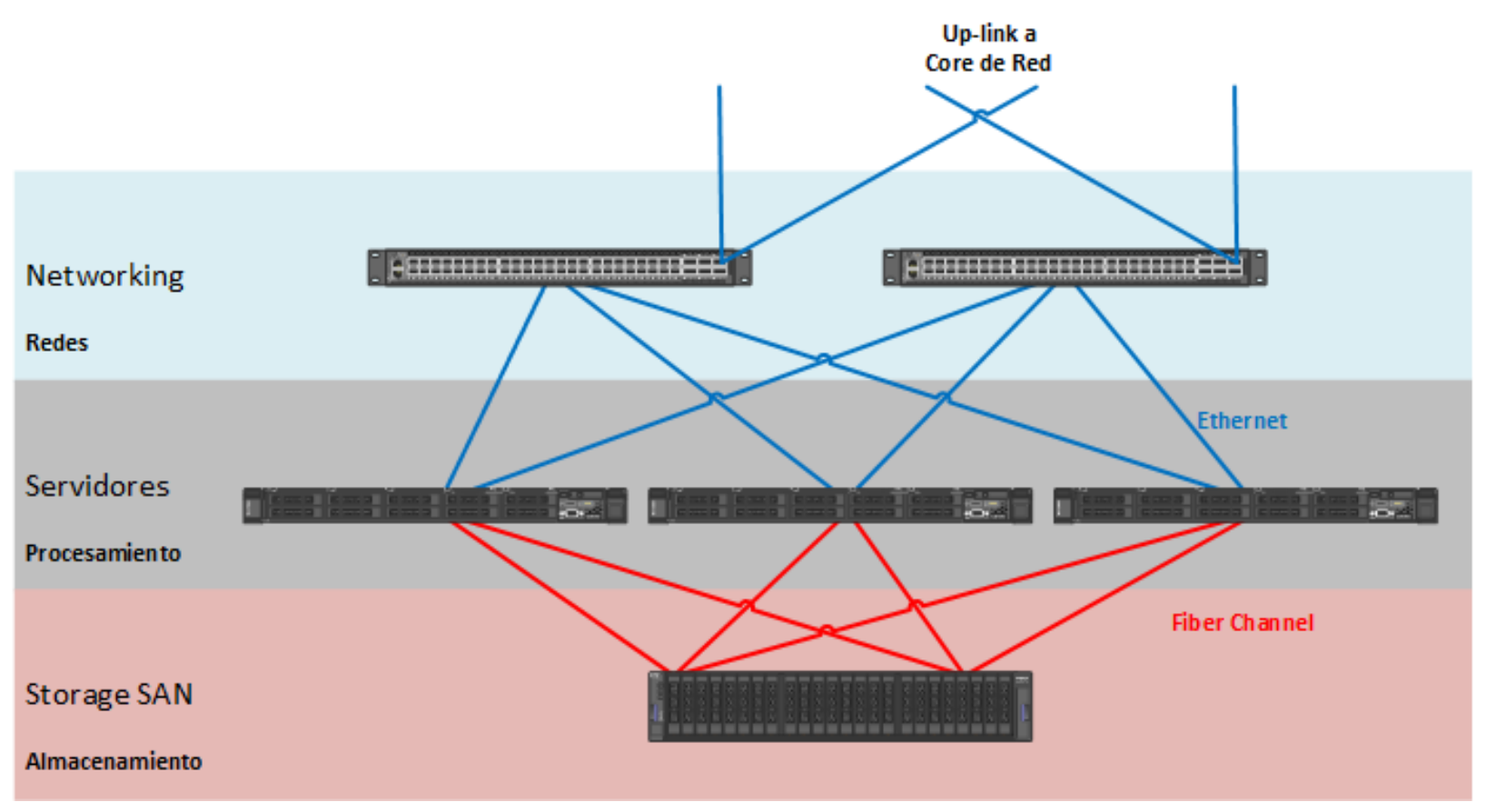

Figura 4. Clúster de servidores con storage SAN compartido. 
Como se observa en el gráfico 4, la infraestructura de hardware deberá considerar redundancia en las conexiones entre los nodos de procesamiento, el almacenamiento y la red. Asimismo, la conexión hacia la red del edificio debe contemplar switches redundantes. Los switches de red pueden ser desestimados en caso de tener puertos disponibles en la infraestructura existente.

En cada servidor se deberá montar un hipervisor para virtualizar los recursos CPU, RAM, Almacenamiento y Redes. Así las cargas que se ejecutan en el clúster podrán migrar de manera inmediata ante eventualidades sin afectar la disponibilidad. Los componentes redundantes y la virtualización de hardware permiten al sistema seguir operando en caso de que un componente falle. La redundancia en el dimensionamiento de los servidores (de acuerdo con recomendaciones del fabricante) deberá contemplar proyecciones de crecimiento, de tal modo que en ningún momento se requiera usar el $100 \%$ de capacidad de los servidores. El clúster debe tener recursos disponibles para garantizar la disponibilidad en ante la falla de al menos 1 nodo.

De acuerdo con la plataforma de virtualización, se debe tener en cuenta las licencias requeridas por el fabricante para el funcionamiento de clúster. Asimismo, las licencias requeridas para los sistemas operativos de las máquinas virtuales que se ejecuten sobre la plataforma. El software también debe contemplar el licenciamiento de las bases de datos requeridas más adelante. De igual forma, el dimensionamiento de éstas últimas dependerá de las recomendaciones del fabricante de BMS.

Es necesario tomar en cuenta las configuraciones necesarias para integrar el clúster a la infraestructura existente. Esta operación demandará horas de servicios para integración a dominios de seguridad, cumplimiento de normas de ciberseguridad y documentación. Asimismo, puede demandar la coordinación para configuraciones de dispositivos de red, reglas de firewall y herramientas de respaldo de información.

\section{Etapa 4. Consenso}

Con la información de cantidad y tipo variables por subsistema, dispositivos de control y sus protocolos, información del edificio y operativa del mismo, arquitectura de hardware definida e integraciones requeridas a otros sistemas se puede realizar el dimensionamiento de los componentes de hardware y software para la solución de BMS tomando en cuenta las consideraciones generales. Esta etapa puede iterar nuevamente el proceso de dimensionamiento de acuerdo con restricciones presupuestarias, brechas de experiencia encontradas o cambios de requerimientos en el sistema.

En esta etapa se concertará el alcance de la solución y los servicios que este conlleve. Se debe tener claro el alcance del sistema y las expectativas de este. Es recomendable poder segmentar el proyecto en fases para 
poder sobrellevar restricciones presupuestarias.

Ejemplo: Arquitectura de solución BMS para un hospital

El objeto de estudio como ejemplo contempla el dimensionamiento del BMS para un hospital. El hospital comprende 2 edificios contiguos, Torre A con 8 niveles en una superficie de aproximadamente $1400 \mathrm{~m}^{2}$ y Torre B con 5 niveles en una superficie de aproximadamente de $2500 \mathrm{~m}^{2}$. Distribuidos se encuentran áreas para atención de emergencias, quirófanos, equipamiento de diagnóstico avanzado, consultorios de diversas especialidades,

maternidad, neonatología, áreas de internación, terapia intensiva, laboratorios, bodegas climatizadas, servicios de lavandería, comedores de personal y de visitantes. Como transporte vertical, ascensores de personal en cada torre y ascensor de servicios en la torre B. Las azoteas de ambas torres se disponen para equipamiento de climatización. Los predios cercanos para el equipamiento eléctrico de generación y transformación eléctrica. Asimismo, almacenamiento de combustible y gases clínicos. Los sistemas de CCTV y Acceso están contemplados de acuerdo con las áreas comunes, equipamiento monitoreado y otras áreas de interés.

Con el objetivo de hacer más explícita la dinámica del dimensionamiento, se presenta brevemente su aplicación en el dimensionamiento de una solución BMS para un hospital. A continuación, se detalla la solución propuesta para un hospital, sus componentes de software, hardware e interacción con los subsistemas del edificio. Los siguientes datos muestran los resultados de la cuarta iteración del proceso.

\section{Etapa 1. Consideraciones generales}

El desarrollo de la arquitectura del BMS tomó cuatro meses, se contó con un equipo multidisciplinario compuesto por un arquitecto de soluciones, especialistas de BMS, servidores, virtualización y telecomunicaciones. Se desarrolló una serie de reuniones en las que se releva información a detalle de las consideraciones del proyecto en cada iteración. Las consideraciones de la primera etapa marcarán el lineamiento de la operativa esperada del edificio, infraestructura de hardware, esquemas de licenciamiento, alcances y expectativas de la solución.

El objetivo inicial del hospital es generar ahorros con la solución BMS a través del control y automatización de comportamientos de los subsistemas de caldera, climatización e iluminación solamente en primera instancia. Se requiere el monitoreo del resto de los subsistemas comprendidos en el edificio.

Los sistemas monitoreados y controlados son: Caldera, Climatización, \|luminación.

Los sistemas monitoreados son: Almacenamiento de combustible, Almacenamiento de gases clínicos, Ascensores, CCTV, Control de acceso, Distribución eléctrica e Hidrosanitario. 
Se requiere almacenar los datos por 3 años en línea para el posterior análisis de información y encontrar tendencias en los consumos de energía de los subsistemas y áreas del hospital. El almacenamiento para 3 años debe ser considerado en la infraestructura. También se consideran las licencias necesarias para la producción de reportes y análisis de datos.

Como lineamiento de hardware, el hospital no tiene una arquitectura de hardware ni marca predefinida. Este escenario (greenfield) es ventajoso ya que permite tener un diseño a medida para la solución.

Esta primera etapa nos brinda el lineamiento básico para la definición de componentes y la operación entre ellos.

\section{Etapa 2. Relevamiento de variables}

Se contempla la integración de monitoreo y control de los subsistemas y variables descritos en la tabla 1.

Tabla 1. Listado de subsistemas y variables

\begin{tabular}{lcccc}
\hline \multirow{2}{*}{ Subsistemas } & \multicolumn{2}{c}{ Variables de entrada } & \multicolumn{2}{c}{ Variables de salida } \\
& Digital & Analógica & Digital & Analógica \\
\hline Almacenamiento de combustible & 4 & 2 & & \\
Almacenamiento de gases clínicos & 7 & & & \\
Ascensores & 48 & 24 & 4 & \\
Caldera & & 16 & & \\
CCTV & 350 & & 429 & \\
Climatización & 721 & 273 & & \\
Control de acceso & 140 & & & \\
Distribución eléctrica & 248 & 885 & & \\
Hidrosanitario & 56 & 1 & $\mathbf{1 8 0}$ \\
lluminación & 317 & & $\mathbf{7 5 0}$ & $\mathbf{2 1 7}$ \\
Total subsistemas & $\mathbf{1 8 9 1}$ & $\mathbf{1 2 0 1}$ & 150 & \\
Reserva 20\% & 378 & 240 & $\mathbf{9 0 0}$ & \\
Total variables & $\mathbf{2 2 6 9}$ & $\mathbf{1 4 4 1}$ & $\mathbf{4 8 2 6}$ & \\
Gran total & & & & \\
\hline
\end{tabular}

La tabla 1 presenta el resumen del relevamiento de los once subsistemas estima 4826 variables, considerando una proyección de crecimiento al $20 \%$. El $77 \%$ de las variables son de monitoreo 0 entrada y sólo $23 \%$ corresponde a variables de control o salida (caldera, climatización e iluminación). Los subsistemas que contemplan más variables son distribución eléctrica, climatización e iluminación.

El tipo y cantidad de las variables son provistas por cada especialista en el subsistema del edificio. Además de las cantidades, proporcionan información de los protocolos utilizados en la red de instrumentación hacia los dispositivos de campo. La cantidad de variables y protocolos utilizados determinan la 
cantidad de licencias de software, protocolos habilitados y horas de servicios requeridos para el dimensionamiento del sistema. Asimismo, proveen información para dimensionar el tamaño de servidores y almacenamiento para el procesamiento de la información del BMS. Finalmente, la cantidad de dispositivos de control afecta la cantidad de puertos de red requeridos en los switches de acceso. Esta última es información es útil para el dimensionamiento de la red de datos del edificio (fuera del alcance del proyecto).

En esta misma etapa se obtienen planos actualizados de los ambientes para la estimación de horas de servicios requeridas para la abstracción los ambientes en el sistema BMS.

\section{Ełapa 3. Diseño de arquitectura}

El diseño requerido por el hospital demanda que la solución BMS incluya un clúster dedicado para garantizar la alta disponibilidad bajo la plataforma VMware.

Se considera el clúster para las cargas de servidores de BMS, base de datos e integración. El clúster albergará 3 máquinas virtuales, cada una de ellas con 8 cores y 16 GB de RAM de acuerdo con el dimensionamiento del fabricante de BMS. Los roles de BMS, base de datos e integración deben ser instalados en servidores independientes. Si bien la separación de roles incrementa los requerimientos de infraestructura, también brinda facilidades en las tareas operativas de mantenimiento y respaldo más adelante. Se considera un clúster pequeño de 3 nodos físicos con un almacenamiento compartido.

La infraestructura de hardware, de acuerdo con el relevamiento, contempla: 1) Almacenamiento SAN con conexión directa al storage a través de Fiber Channel a 16 Gbps. 2) Servidores con procesadores Intel Xeon de 20 cores y 96 GB de RAM. Cada uno con conexiones redundantes a switches Top of the Rack (ToR) a 10 Gbps. 3) Switches ToR con puertos loGbps para la conexión de servidores y puertos para up-link de hasta 40Gbps para up-link hacia el core de red. 


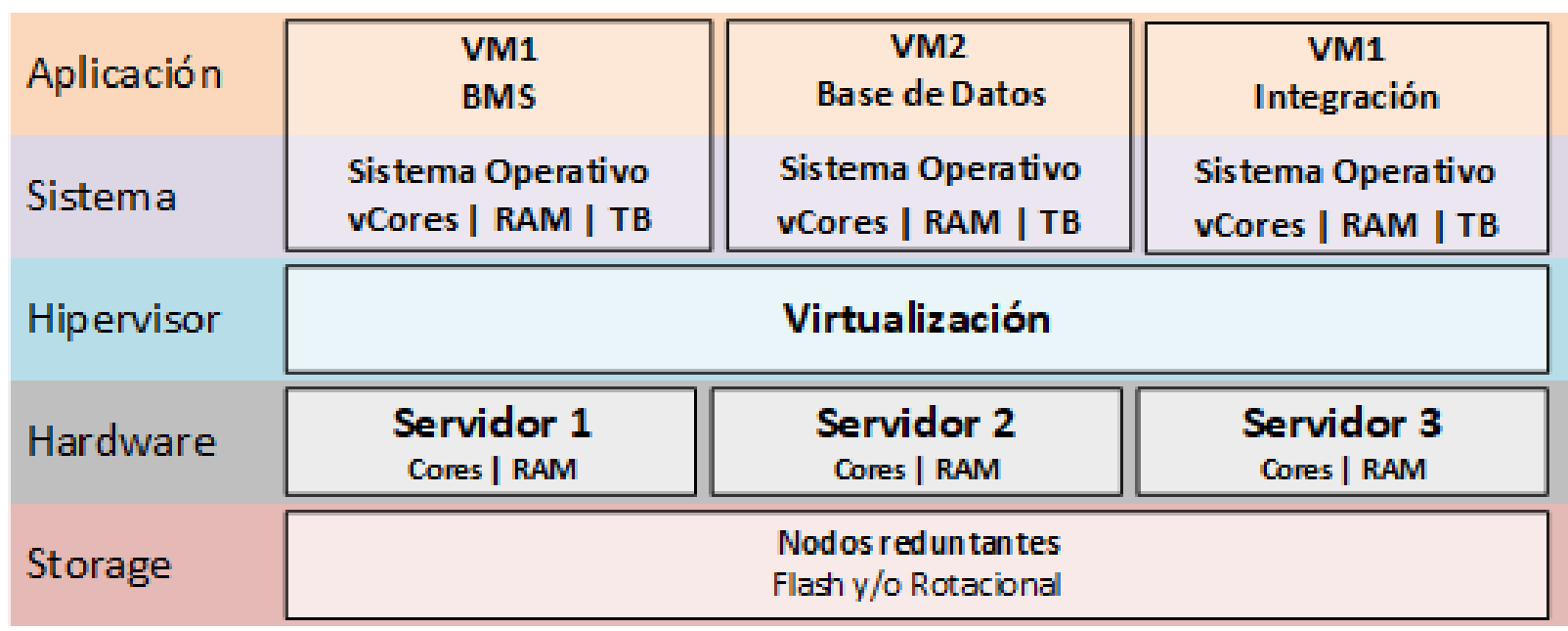

Figura 5. Diagrama de clúster de servidores con storage SAN compartido.

La Figura 5 presenta los componentes de hardware, virtualización, sistemas operativos y aplicaciones.

Sobre la infraestructura de hardware, se contempla la siguiente solución de software 1) VMware como hipervisor habilitando el clúster con 3 nodos, 2) licencias de Windows Server como sistema operativo de las máquinas virtuales para los servidores de BMS, base de datos e integración, 3) licencia de SQL Server Standard para la base de datos de ambos sistemas.

Considerando que el BMS es un sistema crítico, se incluyen contratos de servicio de atención 24/7 para la atención de incidentes

\section{Etapa 4. Consenso}

En esta etapa se validaron todos los componentes de la solución: hardware, software, servicios y soporte, se definieron los montos estimados de implementación del proyecto. En consenso con los interesados el proyecto, se ajustaron los alcances de la solución para viabilizar la adquisición de acuerdo restricciones presupuestarias. Cada iteración produce cambios en la solución que afectan los costos y plazos requeridos para su implementación.

\section{CONCLUSIONES}

Este proceso plantea las consideraciones mínimas requeridas para el generar el dimensionamiento ordenado de cada uno de los componentes para una solución de BMS. Procura simplificar las tareas y ordenar la información para generar un diseño de propuesta claro. Gracias a la aplicación práctica en un hospital se evidenció que, al seguir el proceso, los componentes requeridos para el BMS son supervisados e integrados para garantizar la funcionalidad del sistema en cumplimiento de los requerimientos del hospital.

Este proceso puede asistir en el dimensionamiento para proyectos que requieran una solución completa de 
hardware, software, servicios y soporte para BMS.

En el contexto actual, muchas empresas pequeñas, medianas y grandes, deben elevar la eficiencia, reducir costos e incrementar la productividad para sobrevivir, el principal aporte tiene foco en la economía nacional e internacional y en la salud de sus empresas.

En condiciones normales, los aportes anteriormente descritos generan valor para la comunidad investigativa y empresarial. En condiciones excepcionales, como la crisis actual generada por el COVID-19, se valora mucho más todo aporte al sector empresarial.

\section{REFERENCIAS}

Applied Risk BV. (2019). I Own Your Building (Management System). Amsterdam.

Antesana, Iván Robinson y Medina, Anibal (2016) Sistema de control centralizado de edificios B.M.S. Universidad Católica de Córdoba [Tesis de Grado]

Fundación Giner de los Ríos (2016). Libro Verde. Hacia un sector eficiente. Chile
Gökçe, H; Gökçe, K. (2012) Holistic system architecture for energy efficient building operation

Microsoft (2019) CREATING INTELLIGENT SPACES Five strategies to accelerate smart building transformation. Microsoft Azure - White papers

Osama Omar (2018) Intelligent building, definitions, factors and evaluation criteria of selection. Alexandria Engineering Journal, Volume 57, Issue 4, December 2018, Pages 29032910

Perry, C. (2017). Smart Buildings: A Deeper Dive into Market Segments. Report A1703. (C) American Council for an Energy-Efficient Economy. EEUU

Nguyen, T. (2012). Energy and BuildingsEnergy intelligent buildings based on user activity: A survey. Revista Energy and Buildings. Nijenborgh 9, 9747 AG Groningen, The Netherlands

Van Hoof, B. (2018). DATA-DRIVEN WORK SPACES IOT and AI Expand the Promise of Smart Buildings. Harvard Business School Publishing. MC210190918. EEUU

Vasques, X. (2011). Analysis and knowledge discovery from sensors data to improve energy efficiency Conference Paper . DOI: 10.13140/2.1.2843.4240 